\title{
The Viral Etiologies of Severe Acute Respiratory Infection: Indian Perspective on the Emerging Pathogens
}

\author{
Tushar Shaw* (iD) and V.A. Indumathi
}

Department of Allied Health Sciences, Faculty of Life \& Allied Health Sciences, Ramaiah University of Applied Sciences, MSR Nagar, Bangalore - 560 0054, Karnataka, India.

\begin{abstract}
Severe Acute Respiratory Infection poses a significant threat to human health being a major cause of morbidity and mortality. India has witnessed several outbreaks of different infectious etiologies in the past. Among these, several new viral infections have been classified as emerging threat to humans. The word emerging refers to infectious etiologies that have newly appeared in the community or are rapidly increasing their range, corresponding to upsurge in the number of cases. Several different elements can contribute to the emergence of a new virus disease that may cause epidemic or pandemic around the globe. Containment of these viruses is difficult as most of them are of zoonotic origin. There is no immunity in the community against these viruses leaving individuals vulnerable to the disease. Factors such as socio-cultural, ecological along with human animal interphase creates challenges with respect to the emergence of these viral diseases. The major emerging viral infections of public health importance with respect to severe acute respiratory infection in India has been reviewed in this article.
\end{abstract}

Keywords: Emerging viruses, SARI, COVID-19, public health

*Correspondence: tusharshaw1990@gmail.com; +91 080-45366666

(Received: May 25, 2020; accepted: October 15, 2020)

Citation: Shaw T, Indumathi VA. The Viral Etiologies of Severe Acute Respiratory Infection: Indian Perspective on the Emerging Pathogens. J Pure Appl Microbiol. 2020;14(4):2277-2285. doi: 10.22207/JPAM.14.4.05

(C) The Author(s) 2020. Open Access. This article is distributed under the terms of the Creative Commons Attribution 4.0 International License which permits unrestricted use, sharing, distribution, and reproduction in any medium, provided you give appropriate credit to the original author(s) and the source, provide a link to the Creative Commons license, and indicate if changes were made. 


\section{INTRODUCTION}

Severe acute respiratory infections (SARI) is defined as an acute respiratory illness presenting with fever $\geq 38^{\circ} \mathrm{C}$, cough and shortness of breath in the past 10 days requiring hospitalization ${ }^{1}$. The potential viral pathogens of SARI include seasonal $A$ and $B$ influenza viruses, the new influenza $A$ (H1N1) pdm09 virus strain, human rhinovirus (HRV), human metapneumovirus (HMPV), human adenovirus (HAdV), human parainfluenza viruses (HPIV), respiratory syncytial virus (RSV), human bocavirus (HBoV), enteroviruses and human coronaviruses (HCoVs). Children are prone to get infected with lower respiratory tract infections caused by viruses ${ }^{2}$. These viruses have substantially seen to affect elderly and immunocompromised individuals. In most cases, respiratory viral infections are self-limiting and confined to the upper airways, where they evoke relatively mild symptoms such as sneezing and a runny nose ${ }^{3}$. However, in susceptible individuals, such as newborns and the elderly, the symptoms can impact on the lower airways, resulting in wheeze, shortness of breath, bronchiolitis or pneumonia ${ }^{4}$. The increasingly growing field of molecular diagnostics helps in better understanding of global circulation patterns of respiratory viruses worldwide ${ }^{5}$. Although the pattern of respiratory viruses circulating in the community can be predicted based on their predisposition to specific season ${ }^{6}$ there viruses which may be identified outside their normal seasonal pattern of infection. The similarity in clinical presentation of SARI makes it difficult to identify the underlying cause until a laboratory testing is performed. Therefore in such cases patients are subjected to presumptive treatment ${ }^{7}$. In majority of the centers in India SARI is treated empirically and with supportive therapy due to limited access of laboratory facilities to diagnose the infection. Acute respiratory infections remain one of the leading public health concerns causing high morbidity and mortality specially in low- and middle-income countries where the diagnosis can be challenging. A few of the viruses have caused outbreaks in the recent past and are of global health concern. Similar clinical signs and symptoms are caused by various respiratory viral pathogens; hence, rapid simultaneous identification of different viruses is of major epidemiological and clinical interest. The past outbreaks of the respiratory viruses were itself an indication of their evolving nature. The current review brings in light the evolving epidemiology of the respiratory viruses and their importance in accordance with the Indian perspective.

\section{Changing epidemiology of SARI}

Since 1963, World Health Organization has been instrumental in estimating the actual burden of SARI in the midst of evolution of communicable diseases. A study published in 1978 showed the changes that occurred in 1957/1958 and 1967/1968 in 32 countries at different stages of development-i.e., highly developed, less highly developed, and developing ${ }^{8}$. The mortality rates in the group of highly developed countries were 58.6 and 60.3 per 100000 population in the year 1957/1958 and 1967/1968, respectively. In the less highly developed countries, mortality rates attained 83.6 and 62.1 respectively, and in the developing countries they were as high as 169.4 and per 100000 population, respectively. Since 1963 WHO has organised a system for the collection and dissemination of laboratory and epidemiological information on viral diseases. A study on Global burden of diseases estimated the annual death attributed to major causes of lower respiratory tract infections (S. pneumoniae, Hib, influenza, and RSV) ${ }^{9}$. In children younger than 5 years acute respiratory infections caused $13.1 \%$ of all deaths worldwide. Whereas a mortality of $4.4 \%$ was documented in people of all ages. The same study also reported that India accounts for one third of the death due to lower respiratory infections majorly diagnosed in children younger than 5 years.

\section{Seasonal and Pandemic Influenza}

Influenza viruses belong to the family "Orthomyxoviridae", an RNA virus which presents with varied antigenic characters. The three main types of Influenza virus reported till date are type A, B, and C. Type A and B have been responsible for most of the epidemics and outbreak caused till date by influenza virus. In the last century influenza virus has causes epidemics related to Acute Respiratory Infections (ARI) in every 1-4 years. The first epidemic was documented in early $1694^{10}$. The Spanish flu was one of the greatest pandemics that occurred during 1918 killing more than 21 million people worldwide ${ }^{11}$. Emergence of a novel influenza A (H1N1) (swine origin) virus 
with a combination of new gene segments was reported in $2009^{12}$ and the pandemic was declared to be over by WHO in $2010^{13}$. A cumulative data from $17^{\text {th }}$ February 2020 to 01 March 2020 was reported to WHO from 111 countries, areas, or territories. During this time, the WHO (Global Influenza Surveillance and Response System) laboratories tested 233445 specimens. Among these samples, 62423 were positive for influenza viruses, of which type A was documented in 42013 (67.3\%) cases and 20410 (32.7\%) were influenza B. Of the sub-typed influenza, A viruses, accounted for 7348 (74.5\%) were influenza $A(H 1 N 1)$ pdm09 and 2516 (25.5\%) were influenza $A(H 3 N 2)$. Of the characterized $\mathrm{B}$ viruses, $\mathrm{B}-\mathrm{Victoria}$ lineage was found in 1574 (98.9\%) whereas 18 (1.1\%) belonged to the B-Yamagata lineage.

Influenza A virus has the ability to undergo changes in its surface antigens leading to events called "antigenic shifts" (major changes) and "antigenic drifts" (minor changes). Antigenic shifts lead to epidemics and pandemics of influenza $A$, whereas antigenic drifts have been related to localized outbreaks. These are the major concerns of influenza epidemic and to create target vaccines against the pathogen.

Influenza commonly affects young individuals; however, the mortality and morbidity are observed among individuals with extreme of ages and individuals affected with definite highrisk medical conditions such as cardiovascular diseases and diabetes mellitus ${ }^{14}$. A systematic review and metanalysis reported individuals with diabetes mellitus to be at 2.21 times at higher risk of death ${ }^{15}$. The transmission of influenza virus is through droplet nuclei (larger than $5 \mu$ ) released from individuals infected with the virus ${ }^{16}$. Close contact is usually required for acquiring infection due to the large size of the infectious droplets. The disease shows an acute onset of symptoms following a short incubation of 1-2 days fever headache and malaise accompanied by sore throat and dry cough. The uncomplicated infection may last for 4 to 8 days and eventually subsides. At several instances' influenza can present as pneumonia which may be due to primary influenza virus or caused due to secondary bacterial and viral infections. The clinical manifestations and seasonal outbreak are key signs for early diagnosis of influenza. In complicated cases the diagnosis of influenza requires laboratory confirmation using available tests such as nucleic acid detection or rapid diagnosis kits.

Influenza in India: what the country needs?

The major outbreak of influenza in India during the post pandemic period was in 2015 reporting 42,592 cases and 2990 deaths. The influenza surveillance conducted from 2009 to 2013 reported $6,193(14.0 \%)$ of SARI to be positive for influenza virus ${ }^{17}$. The interesting finding of the study was that despite a varied range of environmental and latitudinal variability across India there were two major patterns of influenza circulating in the country. The seasonality and transmission of the influenza virus has been associated with seasonal fluctuations in the environment and social factors ${ }^{18}$. This has an implementation on strategizing vaccine formulation and correct time best suited for these regions. A modelling study estimating mortality associated with global seasonal influenza predicted a median annual associated mortality of 148105 for low and middle-income countries ${ }^{19}$. From the current data it is well estimated that influenza is a growing health care problem in India. The types and subtypes of strains circulating in the country and the seasonality of annual outbreaks are also known ${ }^{17}$. However, there is a paucity in data with respect to the severity of the disease and its sub classification with respect to hospital admissions and deaths, especially among the high-risk groups. Identifying the burden of influenza among these individual groups will further help to conduct cost effective vaccine trials. During the 2009 pandemic 1.5 million doses of vaccine were imported by the government for vaccination among high risk groups, however the acceptance and utilization of these vaccine were not very satisfactory ${ }^{20-25}$. Despite all the efforts, India recorded 38,730 cases and 2024 deaths by the end of $2009^{26}$. This clearly highlights the lack of awareness prioritizing the need for information, education and communication programmes targeting the general public and health care workers. Additionally, the government should bring in programmes for mandatory vaccination of health care workers who are at highest risk of acquiring the infection. Given the widespread presence of influenza virus in nature, the probability of emergence of a modified influenza virus with a 
high rate of transmissibility among humans might evolve. Uncertainty lies in how India perceive this threat and take action.

Severe acute respiratory syndrome (SARS): the 2002 epidemic

Severe acute respiratory syndrome (SARS) originated in Southern China in November 2002 caused by a novel coronavirus belonging to the Nidovirales order positive-strand RNA viruses) which had spread rapidly around the globe mostly affecting the Asian countries. By the end of the epidemic 8422 individuals were affected by the disease with 916 reported deaths (11\% case fatality $)^{27}$. The disease was associated with serious respiratory symptoms, leading to pneumonia ${ }^{28}$. The origin of SARS- CoV and its potential vector still remains unknown, however genetic studies provide evidence supporting zoonotic origin of the virus $^{29}$. Interestingly genetically and antigenically similar viruses were isolated from different animal sources in China ${ }^{30}$. The novel coronavirus was different from other coronaviruses reported previously showing a variation among the structural proteins $(S, E, M \text {, and } N)^{31}$. First case of SARS was reported from India in April 2003 in an individual with travel history to Singapore and Hong Kong. There were only 3 probable case of SARS reported from India during the 2002-2003 epidemic. Till date there are no confirmatory test for SARS available and PCR test is not validated. As with other viral diseases there is no cure for SARS and to date there are no vaccines available. SARS epidemic was the first infectious disease of the 21st century which newly emerged as a cause of human illness. During the epidemic it was observed that the advanced technologies of the 21st century played a relatively small role in combating the outbreak and the conventional methods of isolation and hygiene practices proved to be more helpful in combating the disease. The absence of new clinical cases suggested that SARS-CoV is no longer present in the population; however, the possibility of clinically "silent" infections or virus carriers in the environment could not be ruled out completely. The outbreak of SARS demonstrates the importance of an emerging infection which can have a considerable impact on economy of the world. Although the impact of SARS 2002 epidemic was not much on
India but it stimulated an emergency response for preparedness of an emerging and epidemic prone disease in near future.

\section{Middle East respiratory syndrome (MERS) coronavirus}

From 2012 to 2019, 2442 MERS-CoV cases were reported from 27 countries with an estimated death of $842^{32}$. Majority of the cases are reported from Africa, the Middle East, and southern Asia where virus is reported to be present in dromedary camels. The spread of "MERS-CoV" infection from human to human has been found among the family members and health care workers. The disease presents with typical signs of pneumonia with incubation ranging from 2-14 days. Severe symptoms are observed among immunocompromised, elderly and individuals with underlying comorbidities ${ }^{33}$. A real time reverse transcriptase PCR was developed by Centers for Disease Control and recommended for detecting MERS-CoV from clinical samples ${ }^{34}$. The disease is known to have a zoonotic origin where in the presence of identical sequences of isolates recovered from a patient and from camel was documented $^{35}$. Despite of this the transmission dynamics of the virus from the zoonotic origin to humans is not well explored. There is no specific treatment or vaccines for MERS CoV. The emergence of MERS CoV was a reminder of 2002 SARS epidemic. Both were due to coronavirus and are thought to be originated from animal sources. The difference lies in their cellular selection for infection. SARS-CoV uses angiotensin-converting enzyme 2 as receptor in the host, whereas MERSCoV recognizes cluster of differentiation $26^{36,37}$.

The global threat of MERS has reduced over time, this was achieved by understanding the knowledge gaps with regard to the spread of the diseases, having an enhanced surveillance and early case detection. The early outbreaks were controlled by proper infection prevention strategies maintained at hospitals and community. In view of international movement between India and Midlle East countries there is always a high risk of a individual bringing in the disease to India. If a MERS CoV infected individual arrives in the country would the nation be able to encompass the infection. Was the MERS-CoV outbreak an alarm to a bigger upcoming threat? 


\section{SARS-CoV-2 the pandemic of coronavirus disease 2019 (COVID-19)}

The SARS-CoV 2 belonging to the coronaviruses family is the etiology behind the ongoing outbreak of coronavirus disease 2019 (COVID-19). COVID-19 was first reported in Wuhan, in the Hubei province of China in December 2019. What started as an outbreak in China soon spread across the world. As of September 2020 there have been 33 million confirmed cases in 213 countries, with 996000 reported deaths. Epidemiology of SARS CoV 2 in different states of India is depicted below (Table 2). Individuals infected with the disease commonly present with fever and cough which might progress to pneumonia. Similar to SARS and MERS the severity of the disease is majorly observed among elderly and individuals with underlying comorbid conditions. The SARS CoV- 2 has shown $88 \%$ sequence similarity to coronaviruses found in bats which indicates its zoonotic origin. The virus has $70 \%$ identity to SARS coronavirus whereas it showed only $50 \%$ identity with MERS coronavirus ${ }^{38}$. The virus shows a high rate of transmissibility that can be attributed to the fact that S protein of SARS CoV-2 can bind human ACE2 receptor with 10-20 folds higher affinity ${ }^{39}$ compared to SARS CoV. There is an uncertainty regarding the crude fatality rate of the disease but it is assumed to be as high as $1-2 \%$ of all infection $s^{40}$. For emerging infectious diseases, the case fatality ratio is an important indicator not only of the disease severity but also for its significance as a public health concern. Despite of the lowercase fatality compared with other respiratory viruses COVID-19 has spread more rapidly.
Structural modelling of SARS CoV-2 identified factors which makes this virus more infectious than other coronaviruses. Coronavirus uses a 'spike' protein to bind to human cell membrane, this process is activated by specific enzymes found in lots of human tissues ${ }^{39}$. This explains some of the symptoms in individuals affected with coronavirus involving organs other than the lungs. Another study from China observed an association between the ABO blood type and COVID-19 ${ }^{41}$. Studies have reported that anti-A antibodies specifically inhibit the adhesion of SARS CoV to ACE2 receptors. The absence of these antibodies among individuals with Blood Group A makes them more susceptible to the disease. However, these results are inconclusive due to lower patient numbers and need further evaluation. In view of high mutation rates coronaviruses are unpredictable and an in depth understanding of its pathogenesis is the current need of the hour. The current diagnosis of the disease depends on reverse-transcription polymerase chain reaction (RT-PCR), targeting the $E$ gene for screening and the RdRp gene for final confirmation ${ }^{42,43}$. However, Sometimes, RT-PCR tends to give false-positive and false-negative results $^{44}$. Evidence from the previous SARS epidemic reveal that serological responses, including virusspecific immunoglobulin, immunoglobulin $M$, and immunoglobulin $\mathrm{G}$, can permit for serological diagnosis ${ }^{45}$. However serological tests have their limitations in view of cross reactivity of antigens used, reducing the specificity of the test. There is no current evidence from randomized controlled trials (RCTs) for recommending any specific treatment for SARS-CoV-2 infection. There is no

Table 1. Comparison of three coronavirus diseases: SARS, MERS and COVID-9

\begin{tabular}{llll}
\hline & SARS & MERS & COVID-19 \\
\hline Epicenter & Guangdong province China & Saudi Arabia & Wuhan, China \\
Year of outbreak & $2002-2004$ & 2012 & 2019 \\
Clinical Presentation & SARI & SARI & SARI \\
No. of cases & 8422 & 2442 & 33 million* \\
Fatality Rate & $10.8 \%$ & $34.5 \%$ & $3.01 \% *$ \\
RO & $2.2-3.6$ & 0.7 & $2-6.47$ \\
Animal reservoirs & Bats & Dromedary camels & Bats \\
Human receptors & Angiotensin-converting & Dipeptidyl peptidase- & Angiotensin-converting \\
for the virus & enzyme 2 & $4 /$ CD-26 & enzyme 2 \\
Treatment & Not available & Not available & Not available \\
\hline
\end{tabular}

*Cases documented till September 2020 
Table 2. COVID-19 INDIA as on: 28 September 2020 (https://www.mohfw.gov.in/)

\begin{tabular}{|c|c|c|c|c|}
\hline SI. No & State & Active Cases & Recovered & Death \\
\hline 1 & Andaman and Nicobar Islands & 188 & 3553 & 53 \\
\hline 2 & Andhra Pradesh & 64876 & 605090 & 5708 \\
\hline 3 & Arunachal Pradesh & 2495 & 6495 & 14 \\
\hline 4 & Assam & 29350 & 139980 & 655 \\
\hline 5 & Bihar & 12827 & 164987 & 888 \\
\hline 6 & Chandigarh & 2303 & 9103 & 147 \\
\hline 7 & Chhattisgarh & 31661 & 72224 & 848 \\
\hline 8 & $\begin{array}{l}\text { Dadra and Nagar Haveli } \\
\text { and Daman and Diu }\end{array}$ & 152 & 2848 & 2 \\
\hline 9 & Delhi & 29228 & 236651 & 5235 \\
\hline 10 & Goa & 5097 & 26460 & 401 \\
\hline 11 & Gujarat & 16633 & 113008 & 3416 \\
\hline 12 & Haryana & 16485 & 105990 & 1307 \\
\hline 13 & Himachal Pradesh & 3657 & 10359 & 175 \\
\hline 14 & Jammu and Kashmir & 18199 & 52859 & 1132 \\
\hline 15 & Jharkhand & 12433 & 66797 & 679 \\
\hline 16 & Karnataka & 104743 & 462241 & 8582 \\
\hline 17 & Kerala & 56786 & 117921 & 677 \\
\hline 18 & Ladakh & 1013 & 3032 & 58 \\
\hline 19 & Madhya Pradesh & 22431 & 97571 & 2207 \\
\hline 20 & Maharashtra & 273646 & 1030015 & 35571 \\
\hline 21 & Manipur & 2359 & 7876 & 64 \\
\hline 22 & Meghalaya & 1480 & 3779 & 43 \\
\hline 23 & Mizoram & 535 & 1373 & 0 \\
\hline 24 & Nagaland & 1042 & 4888 & 16 \\
\hline 25 & Odisha & 35006 & 173571 & 797 \\
\hline 26 & Puducherry & 5239 & 20648 & 513 \\
\hline 27 & Punjab & 18556 & 88312 & 3238 \\
\hline 28 & Rajasthan & 19700 & 107718 & 1441 \\
\hline 29 & Sikkim & 692 & 2146 & 33 \\
\hline 30 & Tamil Nadu & 46341 & 525154 & 9313 \\
\hline 31 & Telengana & 29673 & 156431 & 1107 \\
\hline 32 & Tripura & 5689 & 18959 & 273 \\
\hline 33 & Uttarakhand & 10799 & 35672 & 574 \\
\hline 34 & Uttar Pradesh & 55603 & 325888 & 5594 \\
\hline 35 & West Bengal & 25723 & 216921 & 4781 \\
\hline Total\# & & 962640 & 5016520 & 95542 \\
\hline
\end{tabular}

specific treatment recommended for COVID-19, and the vaccines are still under development. However, evaluation of several antiparasitic and antiviral drugs are under trail but the response in not conclusive. The current strategy again depends on the basic infection control practices including preventive strategies focusing on patient isolation, hand hygiene, social distancing, respiratory hygiene, use of mask, PPE and environmental cleaning.

The Genetic Bottlenecks of RNA Viruses

The RNA viruses are said to evolve rapidly and this phenomenon depends on three main features: a) High mutation rates, b) population dynamics (such as genetic drift due to mutation) or c) by external disturbances (such as population bottlenecks). In nature, the genetic bottlenecks may be very frequent during the transmission of many RNA viruses altering the severity of the disease outbreaks. High mutations can occur due to an error-prone RNA-dependent RNA polymerase (without the proof-reading capability) during viral replication of SARS-CoV-2. The error rate in RNA viruses has a limited mutational tolerability, and 
a small increase in this rate can generate what is known as mutational meltdown which can lead to viral extinction. How true this theory holds for SARS CoV 2 is yet to be discovered.

India's preparedness for the global pandemic Looking at the constant outbreaks of emerging pathogens in the past and limited capacity for rapid identification of such viruses, the Department of Health Research (DHR)/Indian Council of Medical Research (ICMR) took initiative to establish 160 Virus Research \& Diagnostic Laboratories (VRDLs) across the country from 2012-2017. Currently 106 VRDL laboratories have been established. These laboratories are currently instrumental in diagnosing the case of COVID-19 in the country. This already operational platform was able to switch gears to provide countrywide diagnosis for SARS-CoV-2 within a month after discovery of this novel virus. Public health interventions were adopted to control the COVID-19 transmission in India. The most recent laboratory surveillance data from ICMR showed $3.9 \%$ positivity among the tested samples from January to April $2020^{46}$. There has been an upsurge in cases documented from India and till end of September more than fifty lakh individuals were affected by this diseases in the country. Currently there are 713986 total hospital beds available in public health care set ups India, which amounts to 0.55 beds per 1000 population ${ }^{47}$. The number of ICU beds available is also low, both in private as well as public health care centers. According to the estimates from other countries $7-10 \%$ of COVID-19 cases may require critical care support (48). Looking at the vast population of the country, if the number of cases keep increasing there can be a deficit in the health care system to manage the disease.

Signs of evolution and indication to future threat Emerging infections are defined as infection that are rapidly increasing in incidence or geographic range, including previously unrecognized diseases such as that of SARS, MERS and COVID-19. The adaptation of the emerging viruses has driven the outbreak of various infectious diseases in the past. Only two human coronaviruses were well known to cause mild illness in humans before the outbreak of SARS in 2002, The epidemic of SARS and MERS flipped the coin revealing how devastating these viruses can be. The advent of SARS CoV- 2 as a pandemic has thrusted the potential of how devastating the Human coronavirus is, which surprised us with its high transmissibility rate affecting 180 countries and territories around the globe. The zoonotic origins of human coronaviruses define the viral adaptability leading to interspecies transmission. A classic example of this is the modification of SARS Co- $V$ accessory protein ORF8 which is absent in SARS-CoV related bat viruses ${ }^{49}$. Numerous such evidences of mutation and genetic recombination has been observed in the viral evolution ${ }^{50}$. A variety of unknown zoonotic coronaviruses are circulating in the wild. There are plenty of occasions when these zoonotic viruses might evolve and recombine, that might cause the emergence of new infection which can be highly transmissible and/or deadly in humans in future. Reduction of unnecessary contact between animals and humans is of utmost importance to avoid the spillover of such viruses from their natural reservoirs.

These emerging viruses are of key concern and pose global threat due to the following:

1. The great diversity and capacity of rapid evolution

2. Active transmission from animals to humans

3. Lack of immunity in the community against these novel viruses

4. Rapid transmissibility

5. Socio-economic factors including health inequalities within countries

6. Movements of people and goods

7. Potential biothreat

\section{CONCLUSION}

Control of emerging infectious diseases require a national policy dedicated to early detection, rapid response and plan of action. The World Health Organization has made several recommendations for such national responses which are under implementation in India. However, looking at a large population of the country emerging infections like COVID-19 poses a great threat. A meaningful response approach to the problem at the systems level is the need of the hour. The main emphasis should be on strengthening the surveillance, partnership building and research guiding public policies in the near future. 


\section{ACKNOWLEDGMENTS}

None.

\section{CONFLICT OF INTEREST}

The authors declare that there is no conflict of interest.

\section{AUTHORS' CONTRIBUTION}

All authors listed have made a substantial, direct and intellectual contribution to the work, and approved it for publication

\section{FUNDING}

None.

\section{DATA AVAILABILITY}

All datasets generated or analyzed during this study are included in the manuscript

\section{ETHICS STATEMENT}

Not applicable.

\section{REFERENCES}

1. Overview of sentinel systems for hospitalized severe acute respiratory infections (SARI) represented in the weekly EuroFlu surveillance bulletin. http://www. euro.who.int/_data/ assets/pdf_file/0005/186863/ Overview-of-SARI-Surveillance-Systems-final.pdf

2. Denny FW, Clyde Jr WA. Acute lower respiratory tract infections in nonhospitalized children. J Pediatr. 1986;108(5):635-646. doi: 10.1016/S00223476(86)81034-4

3. Zaas AK, Chen M, Varkey J, et al. Gene expression signatures diagnose influenza and other symptomatic respiratory viral infections in humans. Cell Host \& Microbe. 2009;17;6(3):207-217. doi: 10.1016/j. chom.2009.07.006

4. Olenec JP, Kim WK, Lee WM, et al. Weekly monitoring of children with asthma for infections and illness during common cold seasons.J Allergy Clin Immunol. 2010;125(5):1001-1006. doi: 10.1016/j. jaci.2010.01.059

5. Nickbakhsh S, Thorburn F, Von Wissmann B, McMenamin J, Gunson RN, Murcia PR. Extensive multiplex PCR diagnostics reveal new insights into the epidemiology of viral respiratory infections. Epidemiology \& Infection. 2016;144(10):2064-2076. doi: 10.1017/S0950268816000339

6. Benkouiten S, Charrel R, Belhouchat K, et al. Circulation of respiratory viruses among pilgrims during the 2012 Hajj pilgrimage. Clin Infect Dis. 2013;1;57(7):992-1000. doi: $10.1093 /$ cid/cit446

7. Njouom R, Yekwa EL, Cappy P, Vabret A, Boisier P, Rousset D. Viral etiology of influenza-like illnesses in Cameroon, January-December 2009. J Infect Dis. 2012;15;206(suppl_1):S29-35. doi: 10.1093/infdis/ jis573

8. Bulla A, Hitze KL. Acute respiratory infections: a review. Bulletin of the World Health Organization. 1978;56(3):481.

9. GBD 2016 Lower Respiratory Infections Collaborators. Estimates of the global, regional, and national morbidity, mortality, and aetiologies of lower respiratory infections in 195 countries, 1990-2016: a systematic analysis for the Global Burden of Disease Study 2016. The Lancet Infectious Diseases. 2018;18(11):1191.

10. Molineux D. Dr Molineux's. Historical Account of the Late General Coughs and Colds;with Some Observations on Other Epidemic Distempers. Philosophical Transactions, 105-11. doi: 10.1098/rstl.1694.0022

11. Johnson NP, Mueller J. Updating the accounts: global mortality of the 1918-1920" Spanish" influenza pandemic. Bulletin of the History of Medicine. 2002;1:105-115. doi: 10.1353/bhm.2002.0022

12. Novel Swine-Origin Influenza A (H1N1) Virus Investigation Team. Emergence of a novel swineorigin influenza A (H1N1) virus in humans. $N$ Engl J Med. 2009;360(25):2605-2615. doi: 10.1056/ NEJMoa0903810

13. Influenza WP. Report of the WHO pandemic influenza A (H1N1) vaccine deployment initiative. 2012

14. Centers for Disease Control and Prevention. People at high risk for flu complications. https://www.cdc.gov/ flu/highrisk/index.htm

15. Mertz D, Kim TH, Johnstone J, et al. Populations at risk for severe or complicated influenza illness: systematic review and meta-analysis. BMJ. 2013;347:f5061. doi: 10.1136/bmj.f5061

16. Bhat N, Wright JG, Broder KR, et al. Influenzaassociated deaths among children in the United States, 2003-2004. N Engl J Med. 2005;353(24):2559-2567. doi: 10.1056/NEJMoa051721

17. Chadha MS, Potdar VA, Saha S, et al. Dynamics of influenza seasonality at sub-regional levels in India and implications for vaccination timing. PLOS ONE. 2015;10(5). doi: 10.1371/journal.pone.0124122

18. Saha S, Chadha M, Al Mamun A, et al. Influenza seasonality and vaccination timing in tropical and subtropical areas of southern and south-eastern Asia. Bulletin of the World Health Organization. 2014;92(5):318-330. doi: 10.2471/BLT.13.124412

19. Iuliano $A D$, Roguski $K M$, Chang $\mathrm{HH}$, et al. Estimates of global seasonal influenza-associated respiratory mortality: a modelling study. The Lancet. 2018;391(10127):1285-1300. doi: 10.1016/S01406736(17)33293-2

20. Seasonal Influenza (H1N1)- State/UT- wise, Year- wise number of cases and death from 2010 to 2017 (till 31st December 2017). https://ncdc.gov.in/showfile. php?lid=275

21. Holla VN, Borker S. H1N1 newer vaccine usage: Experiences from Karnataka state. Indian J Community Med. 2012;37:134-136. doi: 10.4103/0970-0218.96108

22. Anuja U, Rajasi RS, Ratheesh KH, Vijaya Kumar K, Fettil A. Seroconversion following H1N1 influenza vaccination among health care personnel in South Kerala, India. 
Int J Community Med Public Health. 2017;4:428-432. doi: 10.18203/2394-6040.ijcmph20170267

23. Bhaskar E, Thobias S, Anthony S, Kumar V, Navaneethan. Vaccination rates for pandemic influenza among pregnant women: An early observation from Chennai, South India. Lung India. 2012;29:232-235. doi: 10.4103/0970-2113.99105

24. Hiremath RN, Kotwal SMA, Kunte R, Hiremath S, Basannar D, Bhalla S. Acceptance of vaccination against the 2009 pandemic influenza a among health-care workers in Pune, Maharashtra. Indian Journal of Public Health. 2013;57:48-9. doi: 10.4103/0019-557X.111383

25. Suresh PS, Thejaswini V, Rajan T. Factors associated with 2009 pandemic influenza A (H1N1) vaccination acceptance among university students from India during the post-pandemic phase. BMC Infect Dis. 2011;11:205. doi: 10.1186/1471-2334-11-205

26. Kant L, Guleria R. Pandemic Flu, 1918: After hundred years, India is as vulnerable. Indian J Med Res. 2018;147(3):221-224. doi: 10.4103/ijmr.IJMR_407_18

27. Chan-Yeung M1, Xu RH. SARS: epidemiology. Respirology. 2003;8:S9-14. doi: 10.1046/j.14401843.2003.00518.x

28. Ebihara T, Endo R, Ma X, Ishiguro N, Kikuta H. Detection of human coronavirus NL63 in young children with bronchiolitis. J Med Virol. 2005;75:463-465. doi: 10.1002/jmv.20289

29. Holmes KV. SARS coronavirus: a new challenge for prevention and therapy. J Clin Invest. 2003;111:16051609. doi: $10.1172 / \mathrm{JCl} 18819$

30. Guan $Y$, Zheng BJ, He YQ, et al. Isolation and characterization of viruses related to the SARS coronavirus from animals in southern China. Science. 2003;302:276-278. doi: 10.1126/science.1087139

31. Rota PA, Oberste MS, Monroe SS, et al. Characterization of a novel coronavirus associated with severe acute respiratory syndrome. Science. 2003;300:1394-1399. doi: 10.1126/science.1085952

32. Epidemic and pandemic-prone diseases MERS situation update, May 2019. http://www.emro.who. int/pandemic-epidemic-diseases/mers-cov/merssituation-update-may-2019.html

33. Middle East respiratory syndrome coronavirus (MERSCoV) 20 December 2019 | Q\&A. https://www.who. $\mathrm{int} /$ news-room/q-a-detail/middle-east-respiratorysyndrome-coronavirus-(mers-cov)

34. Lu X, Whitaker B, Sakthivel SK, et al. Real-time reverse transcription-PCR assay panel for Middle East respiratory syndrome coronavirus. J Clin Microbiol. 2014;52:67-75. doi: 10.1128/JCM.02533-13

35. Azhar El, El-Kafrawy SA, Farraj SA, et al. Evidence for camel-to-human transmission of MERS coronavirus. N Engl J Med. 2014;370:2499-2505. doi: 10.1056/ NEJMoa1401505

36. Lu G, Hu Y, Wang Q, et al. Molecular basis of binding between novel human coronavirus MERS-CoV and its receptor CD26. Nature. 2013;500:227-231. doi: 10.1038/nature12328

37. Durai P, Batool M, Shah M, Choi S. Middle East respiratory syndrome coronavirus: transmission, virology and therapeutic targeting to aid in outbreak control. Exp Mol Med. 2015;47(8):e181. doi: 10.1038/ emm.2015.76

38. Lu R, Zhao X, Li J, et al. Genomic characterisation and epidemiology of 2019 novel coronavirus: implications for virus origins and receptor binding. The Lancet. 2020;22:565-574.

39. Wrapp D, Wang N, Corbett KS, et al. Cryo-EM structure of the 2019-nCoV spike in the prefusion conformation. Science. 2020;367:1260-1263. doi: 10.1126/science. abb2507

40. Park M, Thwaites RS, Openshaw PJ. COVID-19: Lessons from SARS and MERS. Eur J Immunol. 2020;50(3):308311. doi: 10.1002/eji.202070035

41. Zhao J, Yang Y, Huang HP, et al. Relationship between the ABO Blood Group and the COVID-19 Susceptibility. medRxiv. 2020. doi: 10.1101/2020.03.11.20031096

42. Corman VM, Landt O, Kaiser M, et al. Detection of 2019 novel coronavirus (2019-nCoV) by real-time RT-PCR. Eurosurveillance. 2020;23:25(3). doi: 10.2807/15607917.ES.2020.25.3.2000045

43. Benrahma H, Diawara I, Smyej I, et al. Epidemiological description and analysis of RdRp, $E$ and $N$ genes dynamic by RT-PCR of SARS-CoV-2 in Moroccan population: Experience of the National Reference Laboratory (LNR)-UM6SS. medRxiv. 2020. doi: 10.1101/2020.06.18.20135137

44. Xiao SY, Wu Y, Liu H. Evolving status of the 2019 novel coronavirus infection: Proposal of conventional serologic assays for disease diagnosis and infection monitoring. J Med Virol. 2020;92(5):464-467. doi: 10.1002/jmv.25702

45. Woo PC, Lau SK, Wong BH, et al. Detection of specific antibodies to severe acute respiratory syndrome (SARS) coronavirus nucleocapsid protein for serodiagnosis of SARS coronavirus pneumonia. $J$ Clin Microbiol. 2004;1;42(5):2306-2309. doi: 10.1128/ JCM.42.5.2306-2309.2004

46. ICMR COVID Study Group, VRDLN Team. Laboratory surveillance for SARS-CoV-2 in India: Performance of testing \& descriptive epidemiology of detected COVID-19, January 22-April 30, 2020. Indian J Med Res. 2020;151(5):424-437. doi: 10.4103/ijmr. IJMR_1896_20

47. Yeolekar ME, Mehta S. ICU care in India-status and challenges. J Assoc Physicians India. 2008;56(R):221.

48. Namendys-Silva SA. Respiratory support for patients with COVID-19 infection. Lancet Respir Med. 2020. doi: 10.1016/S2213-2600(20)30110-7

49. Ge XY, Li JL, Yang XL, et al. Isolation and characterization of a bat SARS-like coronavirus that uses the ACE2 receptor. Nature. 2013;503:535-538. doi: 10.1038/ nature12711

50. Su S, Wong G, Shi W, et al. Epidemiology, Genetic Recombination, and Pathogenesis of Coronaviruses. Trends Microbiology. 2016;24:490-502. doi: 10.1016/j. tim.2016.03.003 